\title{
Hybrid endoscopic approach for submucosal tunneling septum division for Zenker's diverticulum
}

A 71-year-old man was referred for a symptomatic Zenker's diverticulum. The patient had undergone a stapled diverticulotomy 7 years ago. A barium esophagram revealed a 3-cm Zenker's diverticulum with evidence of staples ( $\mathbf{F i g} \mathbf{1} \mathbf{a}$ ). The decision was made to perform a peroral endoscopic myotomy of Zenker's diverticulum (Z-POEM).

A submucosal injection was performed with the intention of creating a bleb above the septum using a combination of normal saline and methylene blue. However, the bleb could not be created because of submucosal scarring and fibrosis ( Fig. 1 b, \Video 1). A mucosal incision was made with a triangle-tip knife (KD 640 L; Olympus, Center Valley, Pennsylvania, USA) using EndoCut Q current, effect 3 , and staples were seen through the incision. A clear submucosal layer could not be exposed because of extensive scarring and fibrosis, and thus tunneling was not possible. The decision was made to perform a standard septotomy (myotomy), which was started with a triangle-tip knife and then continued with a scissor-type endoscopic submucosal dissection (ESD) knife (SB knife, Olympus). A standard septotomy of $1.5 \mathrm{~cm}$ was performed using EndoCut Q current, effect 3, after which a clear submucosal layer was observed (\$ Fig. $1 \mathbf{c}, \mathbf{d}$ ).

The procedure was continued using the Z-POEM technique to ensure complete exposure and dissection of the septum. Tunneling was performed along the esophageal and diverticular sides of the septum using a triangle-tip knife and spray coagulation mode, effect 2 . Once the cricopharyngeal muscle septum was completely exposed, it was dissected for $2 \mathrm{~cm}$ down to the bottom of the diverticulum with a scissor-type ESD knife and EndoCut Q current ( $\triangleright$ Fig. 1 e). The mucosal incision was closed using through-thescope clips (MicroTech, Ann Arbor, Michigan, USA) (• Fig. 1 f). The patient was admitted overnight. An esophagram performed the next day showed no leakage, and the patient was discharged on a soft diet. At follow-up 3 months post-procedure, the patient had complete resolution of the symptoms with no residual symptoms while taking a full diet.

The standard approach to Z-POEM starts with a mucosal incision, submucosal tunneling, septum division (septal myotomy), and mucosal closure [1]. On the other hand, in patients with prior such
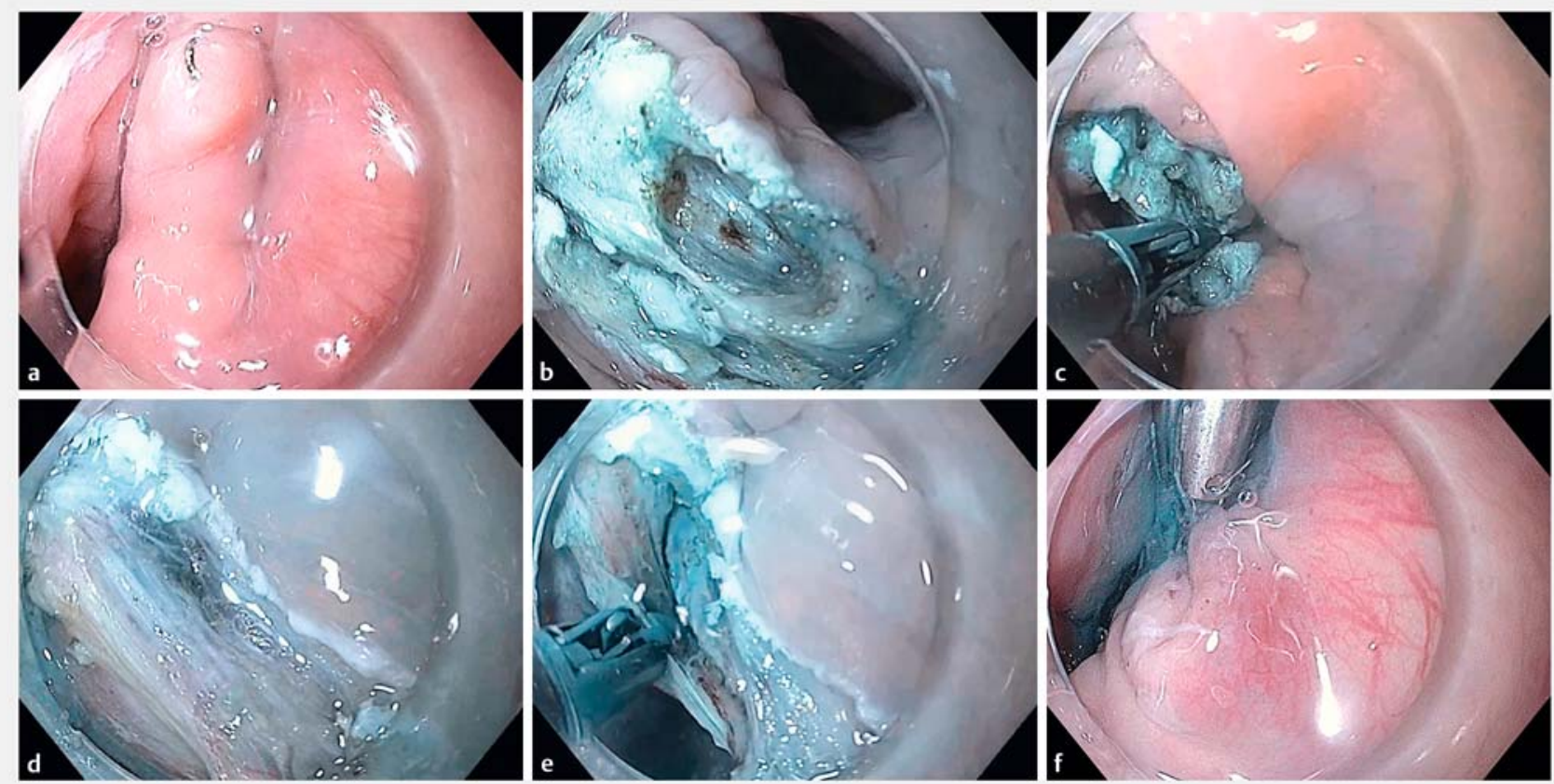

- Fig. 1 Hybrid peroral endoscopic myotomy technique for Zenker's diverticulum (Z-POEM) in a symptomatic 71-year-old man. a A Zenker's diverticulum is identified with visible staples. b After mucosal incision, submucosal scarring was clearly visible. $\mathbf{c}$ A standard septotomy was done by using a scissor-type endoscopic submucosal dissection (ESD) knife. $\mathbf{d}$ After septotomy, the submucosal layer was visualized. e The septum was exposed after submucosal tunneling on both sides of the septum, followed by complete septotomy using the scissor-type knife. $\mathbf{f}$ The mucosal incision was closed using through-the-scope clips. 


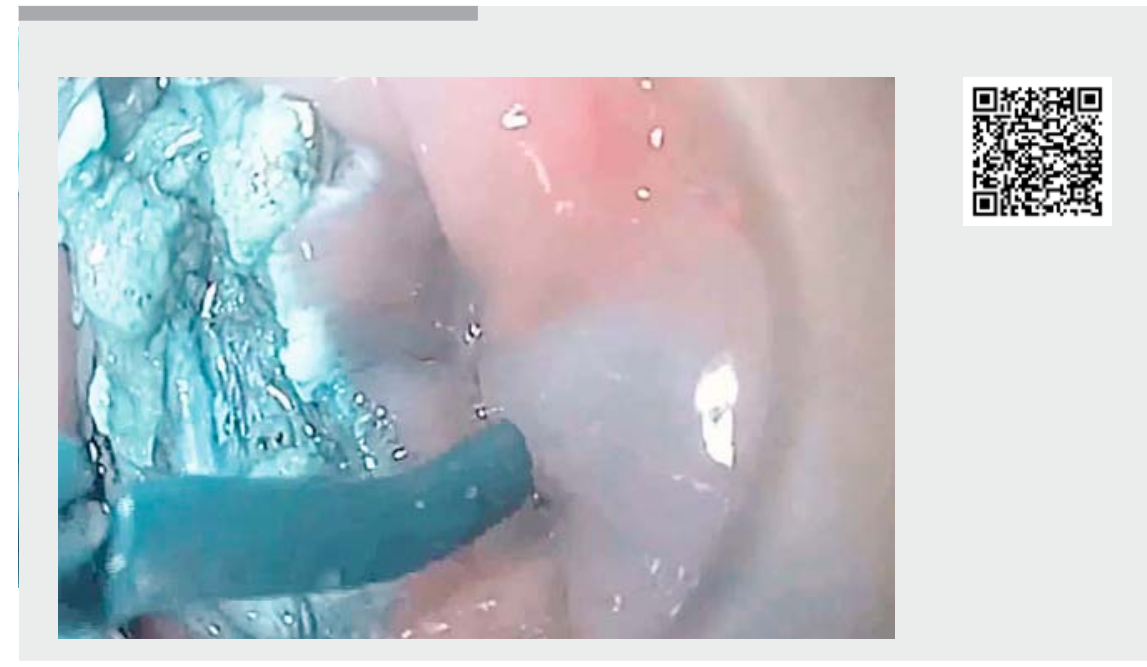

$\checkmark$ Video 1 A symptomatic 71-year-old man with post-surgical fibrosis underwent peroral endoscopic myotomy for Zenker's diverticulum (Z-POEM) using a hybrid approach. Conversion to a hybrid Z-POEM technique when the standard method is found to be not initially possible is a reasonable and effective approach.
- Table 1 Peroral endoscopic myotomy for Zenker's diverticulum (Z-POEM): features and advantages of a hybrid approach.

- The standard Z-POEM technique may not be possible in patients who have had prior interventions

- Starting the procedure with a standard septotomy when Z-POEM is not initially possible and then switching to the Z-POEM technique is a reasonable and effective approach

- Once the submucosal layer can be delineated, this allows for further exposure and dissection of the septum

- The Z-POEM technique with the hybrid approach allows for a complete septotomy, which may reduce the risk of recurrence interventions, a hybrid approach may be necessary. This hybrid approach consists of five steps: (i) mucosal incision, (ii) standard septotomy until a clear submucosal layer is visualized, (iii) submucosal tunneling, (iv) septum division, and (v) mucosal closure) ( $\triangleright$ Table 1 ).

In conclusion, the hybrid Z-POEM technique is safe and feasible in patients with symptomatic recurrent/residual Zenker's diverticulum and submucosal fibrosis from prior interventions. This is the first case to report the use of the Z-POEM technique with the hybrid approach in the case of post-surgical fibrosis.

Endoscopy_UCTN_Code_TTT_1AO_2AD

\section{Competing interests}

Mouen A. Khashab is a consultant for Boston Scientific, Medtronic, and Olympus. None of the other authors have any conflict of interest to declare.

\section{The authors}

Arunkumar Krishnan, Yervant Ichkhanian, Thomas M. Runge, Mouen A. Khashab Division of Gastroenterology and Hepatology, Johns Hopkins Hospital, Baltimore, Maryland, USA

\section{Corresponding author}

\section{Mouen A. Khashab, MD}

Division of Gastroenterology and

Hepatology, Johns Hopkins Hospital, Sheikh Zayed Building, 1800 Orleans Street, Suite

7125G, Baltimore, MD 21287, USA

Fax: +01-443-683-8335

mkhasha1@jhmi.edu

\section{Reference}

[1] Yang J, Novak S, Ujiki M et al. An international study on the use of peroral endoscopic myotomy in the management of Zenker's diverticulum. Gastrointest Endosc 2020; 91: 163-168
Bibliography

Endoscopy 2020; 52: E457-E458

DOI 10.1055/a-1164-7569

ISSN 0013-726X

published online 12.5 .2020

(C) 2020. Thieme. All rights reserved.

Georg Thieme Verlag KG, Rüdigerstraße 14,

70469 Stuttgart, Germany

\section{ENDOSCOPY E-VIDEOS \\ https://eref.thieme.de/e-videos}

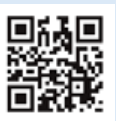

Endoscopy E-Videos is a free access online section, reporting on interesting cases and new techniques in gastroenterological endoscopy. All papers include a high quality video and all contributions are freely accessible online.

This section has its own submission website at https://mc.manuscriptcentral.com/e-videos 